\title{
PAPILLARY CYSTIC NEOPLASM OF THE PANCREAS: A RARE PRESENTATION
}

\author{
Mirko Žganjer, Anto Pajić, Ante Čizmić, Irenej Cigit, Božidar Župančić
}

Children's Hospital Zagreb, Croatia: Department of Pediatric Surgery

\begin{abstract}
Summary: Papillary cystic neoplasm is a rare tumor and is usually found in young female patients. Procedure: Here we describe a rare case of PCN in 16year old girl, which was presented with abdominal pain in last 3 years. Preoperative diagnosis was suggested by ECHO and CT of the abdomen. The sonographic examination of the abdomen showed a tumor measuring $6,5 \times 5,5 \mathrm{~cm}$ in diameter in epigastrium. It was excised totally. Histological description confirmed the diagnosis of PCN. The patient is currently in good health without signs of relapse 3 years after surgery. Discussion: Our patient is one of the very few adolescent patients with PCN who was treated successfully with surgery. ECHO and CT of the abdomen suggested the diagnosis which was confirmed by histopatological examination. Conclusion: The diagnosis of PCN is suspected by ECHO and CT of the abdomen and confirmed by specific histologic features. Prognosis is good and tumor should be excised whenever detected.
\end{abstract}

Key words: Papillary cystic neoplasm of the pancreas (PCN); Female; Ultrasound; Computerised tomography of the abdomen; Histological features; Excision

\section{Introduction}

Papillary cystic tumor of the pancreas represents a distinct and unusual clinicopathologic entity. Tumor was described for the first time by Frantz in 1959 (3). About 200 cases have been reported since then, most of them occuring in young females;only a few male patients have been reported (1). Most of the pancreatic neoplasm among children are endocrine (beta -cell tumors) (5). At least 10 types of primary nonendocrine neoplasm have also been described (7) among which papillary-cystic neoplasm is notable because of its rarity and low grade malignant potential. We report a rare case of PCN in a 16 year old girl, that was totally excised with no recurrence on 3 years follow up.

\section{Case report}

A 16 year old girl was admitted to our hospital because of intermitent abdominal pain lasting for 3 years. Vital signs were normal. Physical examination revealed tenderness in epigastric region with palpable abdominal mass without any other sugestive physical findings. Routine laboratory examination (peripheral blood smear, urinalysis and serum electrolytes) were normal. Abdominal ultrasound showed the presence of a lesion in a head of pancreas $6,5 \mathrm{~cm}$ in diameter. The border was well defined and the content displayed a markedly heterogeneous echo structure with scatterd hypoechoic regions. CT showed round lesion $6 \mathrm{~cm}$ in diameter with low density structure and with high density ring located in the head of the pancreas. There was no evidence of metastatic disease to the liver. At laparotomy a well encapsulated tumor growing from the head of the pancreas was found. Excision was performed and all excision margins were free of the tumor without significant damage to normal pancreatic tissue. Postoperative recovery was uneventful with amylase and lipase levels normalizing within 7 days. The tumor was $6,5 \mathrm{~cm}$ in diameter with a smooth external surface and well defined capsule. On cut section the tumor was friable and showed extensive areas of necrosis. Histology revealed a tumoral lesion formed by cells arranged in pseudopapilary pattern with cystic spaces. The cells were cuboidal or cylindrical, small to medium, polygonal in shape with ovoid nuclei with very rare mitotic figures.

\section{Discussion}

Papillary cystic neoplasm of the pancreas occurs predominantly in girls and young women in their second or third decade of life (2). Microscopically it had predominantly papillary arrangement mixed with solid areas. The solid areas are composed of small and medium size tumor cells, which had no obvious atypia. Pseudopapillary structures were found in the cystic degeneration areas. Three different patterns can be recognised, which can affect the imaging information and cause several problems of diffe- 


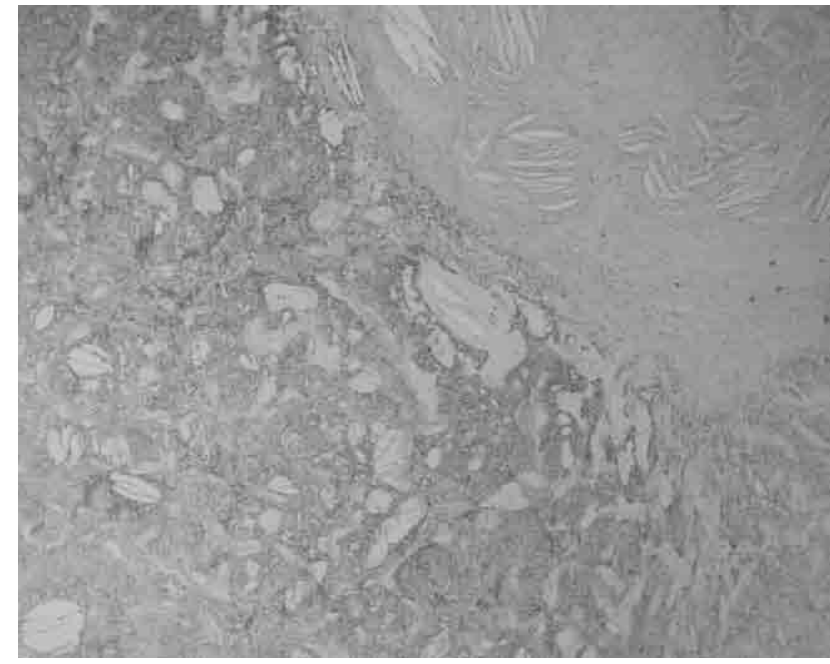

Fig. 1: Tumoral lesion formed by cells arranged in pseudopapillary pattern with cystic spaces.

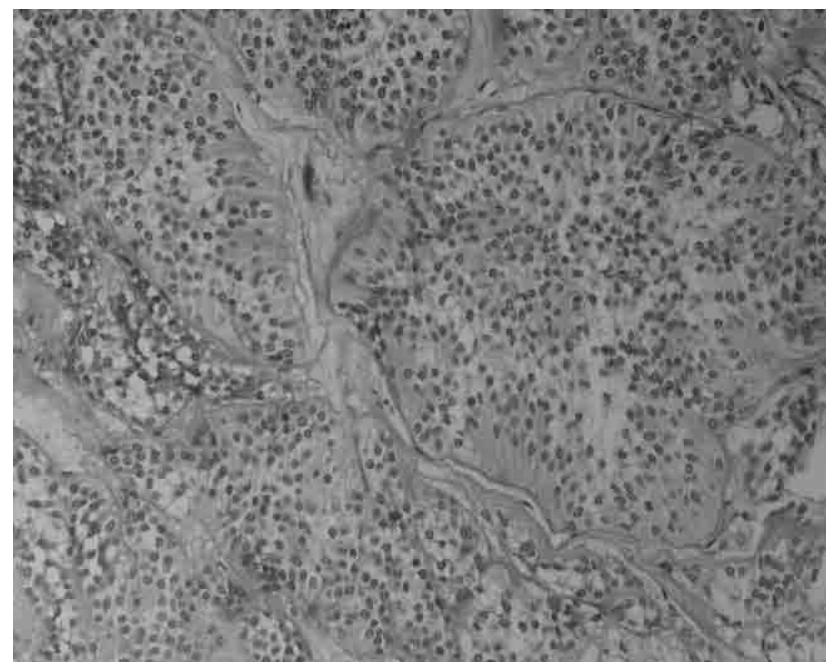

Fig. 3: Tumoral cells with very rare mitotic figures.

rential diagnosis, the masses with completely solid content, the masses with fully cystic content and the masses with mixed content have been the most frequent type of lesion characterised by the presence of solid and cystic areas. Based on high rates of positivity for markers of various pancreatic types (neuron specific enolase, alpha 1 antitrypsin, antichymotrypsin) it has been suggested that the tumor originates from pancreatic pluripotential embryonic stem cells (2). Ultrasonography demonstrates a solid mass, well demarcated and containing cystic areas of variable number and size. On CT the mass is well delineated, nonhomogenous of uneven soft tissue density with central necrosis (8). Papillary cystic neoplasm is a low grade malignancy; metastases are rare, although local spread may occur with tumor rupture (9). Only 5 children with metastasis and/or local

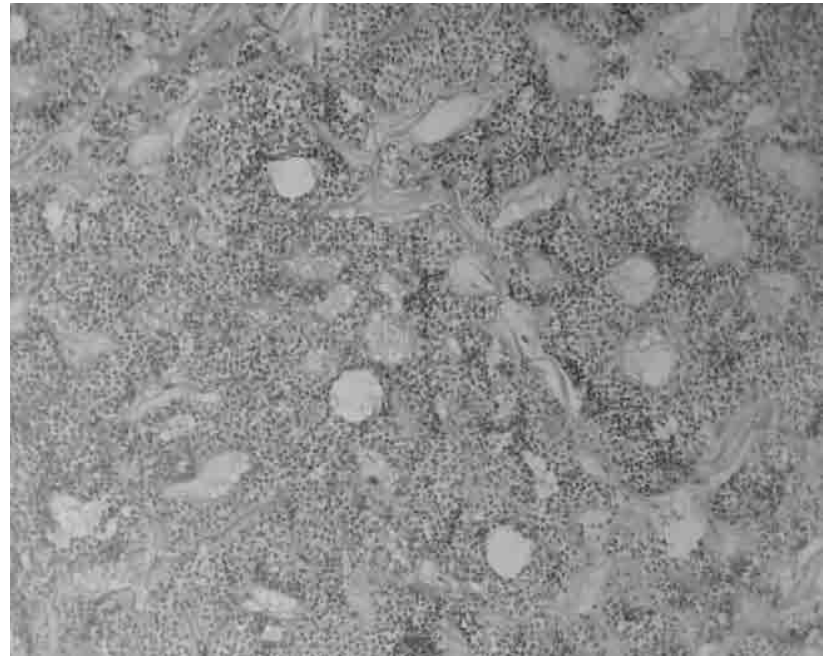

Fig. 2: Tumoral cells were cuboidal or cylindrical, small to medium, polygonal in shape with ovoid nuclei.

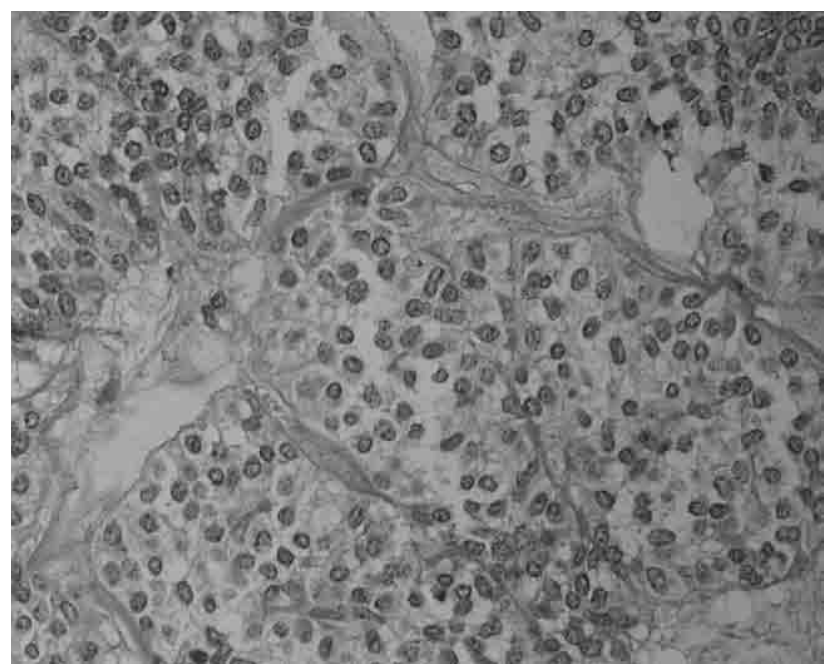

Fig. 4: Tumoral cells with very rare mitotic figures.

recurrences of papillary cystic neoplasm of the pancreas have been reported in the literature (10). Differential diagnosis of papillary cystic neoplasm of the pancreas includes: serous cystadenoma, mucinous cystic neoplasm, nonhyperfunctioning islett cell tumor and pancreatoblastoma. Pancreatoblastoma, the most common form of pancreatic neoplasm in children, may also present with similar appearance but is a more aggresive tumor often with hepatic metastases at the time of diagnosis (4). Treatment of papillary cystic neoplasm is surgical. Complete resection result in cure in the great majority of cases. Since resection of the tumor provides a cure, and there exists a danger of liquid spilage during a biopsy, preoperative percutaneous biopsy is unnecessary (9). Our patient presentation with a palpable abdominal mass is typical for this tumor. 


\section{Conclusion}

Papillary cystic neoplasm of the pancreas is very rare in children. The major presenting symptoms were abdominal pain and abdominal mass. Serum tumor markers showed normal results. Sonography and CT examination showed that the tumor was a heterogeneous mass with solid and cystic components. The tumor was $6,5 \mathrm{~cm}$ in diametar with a smooth external surface and well defined capsule. On cut section the tumor was friable and showed extensive areas of necrosis. Histology revealed a tumoral lesion formed by cells arranged in pseudopapilary pattern with cystic spaces. The cells were cuboidal or cylindrical, small to medium, polygonal in shape with ovoid nuclei with very rare mitotic figures. No metastasis or recurrence was noted during followup (6). Our study confirmed that papillary cystic neoplasm of the pancreas is low-grade malignant tumor. Surgical resection of the tumor is the mainstay of effective management.

\section{References}

1. Chao HC, Kong MS, Lin SJ et al. Papillary cystic neoplasm of the pancreas in children: report of three cases. Acta Paediatr Taiwan 2000;41:101-5.

2. Cubilla AL, Fitzgerald PJ Morphologic patterns of primary non endocrine human pancreas carcinoma. Cancer Res 1975;35:2234-48.
3. Frantz VK. Tumors of the pancreas. Atlas of tumor pathology, $1^{\text {st }}$ series, fascicle. Washington DC: US Armed Forces Atlas of Pathology, 1959:27-8.

4. Horisawa M, Niinomi N, Sato T et al. Frantzs tumor(solid and cystic tumor of pancreas) with liver metastasis. Succesful treatment and long term follow up J Pediatr Surg 1993;30:724-6.

5. Kloppel $\mathrm{G}$ et al. Solid cystic tumor of the pancreas in men. Virch Arch Pathol 1991;418:179-83.

6. Lumkin B, Anderson MW, Ablin DS, Mc Gahan JP. CT, MRI and collor Doppler ultrasound correlation of pancreatoblastoma: a case report. Pediatr Radiol 1993; 23:61-2.

7. Rebhandl W, Felberbauer FX, Paya K. Solid pseudopapillary tumor of the pancreas (Frantz tumor) in children; report of four cases and review of the literature. J Surg Oncol 2001;76(4):289-96.

8. Sabatino D, Kosuri S, Quiles R. Solid and papillary epithelial neoplasm of the pancreas in an 11-yaear-old girl: case report and literature review. Pediatr Hematol Oncol 2003;20(5):357-60.

9. Saw HP, Ho Ml, Chen JY. Solid cystic pseudopapillary tumor of pancreas:report of one case. Acta Paediatr Taiwan 2003;44(6):368-71.

10. Stomner P, Kraus J, Stolte M, Giedl J. Solid and cystic pancreatic tumors. Clinical, histocemical and electron microscope features in ten cases. Cancer 1991;67:1635-41.

Submitted May 2004.

Accepted January 2005.

Mirko Žganjer, M.D., Pančićeva 7, 10000 Zagreb,

Croatia. e-mail: mirko.zganjer@zg.htnet.hr 